\title{
Glottopol
}

Revue de sociolinguistique en ligne

$33 \mid 2020$

Varia - Liberté, égalité, diversité !

\section{Trajectoires de la surdité en France. Parents entendants d'enfants sourds : questionner la fragmentation de l'identité sourde}

\section{Pauline Rannou}

\section{(2) OpenEdition \\ Journals \\ Édition électronique \\ URL : https://journals.openedition.org/glottopol/553 \\ DOI : $10.4000 /$ glottopol.553 \\ ISSN : 1769-7425 \\ Éditeur}

Presses universitaires de Rouen et du Havre

Référence électronique

Pauline Rannou, «Trajectoires de la surdité en France. Parents entendants d'enfants sourds:

questionner la fragmentation de l'identité sourde », Glottopol [En ligne], 33 | 2020, mis en ligne le 01

janvier 2020, consulté le 02 octobre 2021. URL : http://journals.openedition.org/glottopol/553 ; DOI :

https://doi.org/10.4000/glottopol.553 


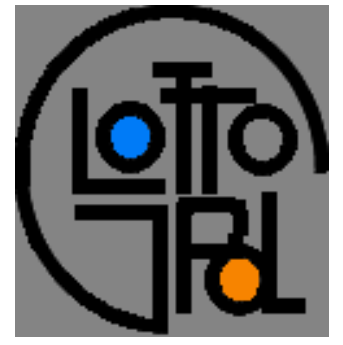

\section{GLOTTOPOL}

Revue de sociolinguistique en ligne n`33 - février 2020

Varia : Liberté, égalité, diversité !

\section{SOMMAIRE}

Hommages à Jean-Baptiste Marcellesi : À ringrazià vi, par Niculau Sorba ; "Ciao Ziu ! » par Philippe Blanchet.

Clara Mortamet : Présentation du numéro : Liberté, égalité, diversité !

Manon Him-Aquilli : "Prendre la parole sans prendre le pouvoir ». La mise en registre de l'horizontalité dans les assemblées générales anarchistes/autonomes.

Siham Hocini : La formulation du désaccord dans les débats télévisés algériens : quels "effetsde-politesse "?

Pauline Rannou: Trajectoires de la surdité en France. Parents entendants d'enfants sourds: questionner la fragmentation de l'identité sourde.

Catherine Combaz-Champlaine: Les rectifications orthographiques de 1990 comme révélateurs du rapport des enseignants à l'orthographe.

Marie-Madeleine Bertucci : Éléments d'épistémologie pour l'étude des espaces ségrégués des banlieues urbaines sensibles de la région parisienne.

Élise Gandon: Usages du numérique et illectronisme: nouvelles tâches pour le lecteurscripteur, nouveaux enjeux d'apprentissage.

Marc Arabyan : Quelle langue pour la recherche neurolinguistique française?

José R. de Arellano : Le rapprochement entre l'espagnol et le portugais pendant la décennie dorée sud-américaine : une problématique de l'horizontalité supercentrale.

Adam Wilson: Normes interactionnelles globalisées et communautés de pratique discontinues : les dynamiques sociolinguistiques du tourisme international.

Mélanie Lancien : Caractérisation de la variation liée à la situation de communication : apport de l'acoustique à la phonostylistique.

Assémou Maurice Ludovic Assémou : La prononciation du français parlé en Côte d'Ivoire.

http://glottopol.univ-rouen.fr 


\title{
TRAJECTOIRES DE LA SURDITÉ EN FRANCE. PARENTS ENTENDANTS D'ENFANTS SOURDS : QUESTIONNER LA FRAGMENTATION DE L'IDENTITÉ SOURDE
}

\author{
Pauline Rannou \\ Université de Moncton
}

\section{Introduction}

En France, la période qui s'étend de la fin des années 1970 à aujourd'hui marque une évolution importante dans la mobilisation pour la reconnaissance de la communauté Sourde ${ }^{1}$ et de la langue des signes française. Le mouvement du Réveil Sourd ${ }^{2}$ (Minguy, 2009) émerge à la suite de la création des Deaf Studies aux États-Unis, qui se concentrent entre autres sur les notions culturelles et linguistiques de la surdité. L'objectif des Deaf Studies est de présenter un champ d'étude interdisciplinaire capable d'apporter des réponses scientifiques aux questionnements et revendications, notamment depuis la publication des travaux de Stokoe (1960) en linguistique, ayant permis de démontrer le statut de langue de la langue des signes américaine. Une décennie plus tard, le Réveil Sourd constitue le point de départ d'une mobilisation pour la reconnaissance de la langue des signes et des communautés Sourdes en France.

Fréquemment employées en opposition avec une vision organiciste de la surdité, l'identité Sourde et la culture Sourde renvoient à l'idée d'une "communauté linguistique », d'un « groupe minoritaire » (Stokoe, Casterline, Croneberg, 1965 ; Padden, 1980), s'opposant à une représentation biomédicale répandue, liée à une déficience audiologique à corriger (Meynard, Delaporte, 2002). Par sa discrimination, et même sa tentative d'extermination, la langue des signes est considérée par le sociologue Bernard Mottez (1970) comme une langue minoritaire et minorisée.

Cet article propose de questionner, dans ce contexte, l'apparente absence des parents entendants et plus largement la place des entendants au sein de la communauté Sourde et des discours relatifs aux notions de culture et d'identité Sourde.

\footnotetext{
${ }^{1}$ La désignation «Sourd» avec majuscule, proposée pour la première fois en 1972 par James Woodward à l'Université Gallaudet aux États-Unis, marque la distinction entre les approches culturelles et audiologiques de la surdité. Le S majuscule indique l'appartenance des Sourds à une « réalité sociologique et linguistique déterminée » (Mottez, $1996: 110)$.

${ }^{2}$ Le Réveil Sourd est le nom donné par l'écrivain André Minguy au mouvement militant d'éveil des Sourds en France à la fin des années 1970. Les Sourds revendiquent une éducation bilingue incluant la langue des signes dans leurs écoles. Ce mouvement aboutira en 1979 en France à la mise en place de l'association « Deux Langues Pour une Education » (2LPE).
} 
Les témoignages présentés sont issus de notre recherche doctorale, menée entre 2014 et 2018. Ils donnent à voir, à travers la confrontation de discours de parents entendants et de sourds adultes en France, des situations de tensions provoquées par un sentiment d'illégitimité, décrit par des parents interrogés, à entrer dans ce qu'ils nomment la communauté Sourde. Les récits portent également sur l'idée d'une évolution nécessaire concernant une représentation essentialiste de la culture et de l'identité sourde. Cette évolution offrirait, selon les personnes rencontrées relatant ce discours, la possibilité aux parents entendants d'être considérés en tant que membres de la communauté sourde et non plus invisibilisés d'une communauté dont leur enfant ferait par essence partie.

\section{Méthodologie de la recherche}

Au total, soixante-et-un entretiens semi-directifs ont été menés dans le cadre de cette recherche, organisée en deux volets ${ }^{3}$. Le premier volet concerne le parcours de parents entendants à la naissance de leur enfant, depuis ce que nous nommons «la première annonce » par les médecins, jusqu'aux choix linguistiques et de scolarisation. Cette première annonce, qui amorce celle de la surdité de leur enfant généralement en maternité, concerne la notification d'un écart audiologique et la nécessité d'effectuer des tests plus approfondis. Cette étape relatée dans les entretiens conditionne de manière importante la relation entre les professionnels et les parents et conditionne également les choix linguistiques des parents par la suite. Dans ce premier volet, douze entretiens ont été menés auprès de parents entendants et sept auprès de personnes sourdes adultes en France.

Les entretiens menés auprès des parents ont eu lieu en intégralité en France et concernent des familles dont les enfants avaient entre quatre et dix ans au moment de l'échange. Les entretiens sont semi-directifs et concernent le parcours de ces familles, à partir des premiers doutes sur la surdité de leur enfant, que ces doutes aient été formulés par les parents eux-mêmes ou leur entourage, avant la mise en place systématique du dépistage néonatal de la surdité en France, ou qu'ils aient été exprimés par les équipes soignantes, au moment du dépistage en maternité ${ }^{4}$, aujourd'hui effectué à deux jours de vie en France. À la suite des premiers doutes, différents tests sont effectués, entrainant la confirmation, ou non, d'un diagnostic de surdité. La trame d'entretien mise en place pour ce volet 1 est divisée en différents thèmes, qui exposent des étapes cruciales dans le parcours de vie de ces familles : dépistage de la surdité, diagnostic et annonce, information par les équipes soignantes et les équipes médico-sociales, choix linguistiques et leurs conséquences, choix de scolarisation, représentations liées à la surdité.

Les entretiens réalisés auprès des personnes sourdes adultes, menés en France également, ont été effectués en langue des signes française, que nous pratiquons, et/ou sous la forme vocale, en fonction des préférences des personnes interrogées. La trame mise en place diffère sensiblement, mais permet une complémentarité dans l'analyse des témoignages. Il s'agit en

\footnotetext{
${ }^{3}$ Le second volet de notre étude propose un regard croisé sur les modèles de scolarisations mis en place pour les élèves sourds en France et en Amérique du Nord, aux États-Unis principalement. 31 entretiens ont été menés dans le cadre du volet 2 de notre recherche. Pour cet article, nous faisons référence au volet 1 mené auprès de parents entendants et de personnes sourdes adultes.

${ }^{4}$ Arrêté du 3 novembre 2014 relatif au cahier des charges national du programme de dépistage de la surdité permanente néonatale : «Le dépistage de la surdité chez le nourrisson a pour objectif la mise en place précoce de prises en charge adaptées pour favoriser le développement du langage et la communication de l'enfant sourd au sein de sa famille, sans préjuger de l'approche éducative qui sera choisie ultérieurement par la famille. La première étape du dépistage consiste en une vérification de l'audition au cours du séjour en maternité par des méthodes objectives, non invasives. Son principal enjeu est, dans un souci d'équité, de rendre le dépistage accessible à tous les nouveau-nés » : https://www.legifrance.gouv.fr/affichTexte.do?cidTexte=JORFTEXT000029754753
} 
effet pour l'ensemble des personnes sourdes interrogées, d'enfants de parents entendants. Les thèmes évoqués concernent le parcours familial, l'aspect audiologique de la surdité, le parcours linguistique, ainsi que le parcours de scolarisation, le parcours professionnel puis les représentations liées à la surdité.

\section{Regard sur l'approche déficitaire de la surdité : une stratégie de l'illusion}

Dans l'approche dite «déficitaire », la surdité est définie en tant que déficience auditive, mise en rapport sur une échelle de valeur dépréciative à la norme des personnes entendantes jugées saines. Les sourds sont victimes d'une pathologie et deviennent des patients à soigner, futurs candidats à une chirurgie réparatrice censée les amener au plus près d'un idéal d'audition. L'objectif consiste, en ce qui concerne la modalité vocale, à réduire au maximum l'écart entre la parole vocale de la personne sourde, parfois désignée en tant qu'accent sourd et celle d'un modèle entendant, considéré comme la norme à atteindre.

Ce témoignage d'une mère entendante interrogée illustre cet «accent sourd », assimilé à un défaut.

Marie : « on s'est vraiment reposés sur le canal auditif parce qu'elle a une très bonne réhabilitation $\downarrow$ les gens qui la voient comme ça / sans voir les appareils pensent qu'elle n'est pas sourde / parce qu'elle parle super bien // enfin elle parle / je veux dire elle n'a pas d'accent $\downarrow$ elle fait des erreurs de syntaxe / de vocabulaire / un peu comme les enfants de son âge mais // elle n'a pas l'accent d'un sourd et en compréhension elle fait souvent illusion $\downarrow$ c'est-à-dire que des fois elle comprend des fois elle ne comprend pas mais elle fait comme si $\downarrow$ ».

Marie est la mère d'Élise, implantée à l'âge de onze mois, qui au moment de notre entretien a six ans. Avant ses onze mois, Élise était appareillée mais les appareils auditifs ne permettant pas une réhabilitation auditive jugée "efficace », ses parents, en concertation avec l'équipe médicale, font le choix de l'implantation cochléaire ${ }^{5}$.

Le témoignage de Marie souligne les préoccupations liées à un écart plus ou moins important à la référence audiologique et les attentes exprimées par des parents d'enfants sourds interrogés dans le cadre de cette étude ayant fait le choix de l'implantation cochléaire. L'accent sourd renvoie à la déficience, au corps handicapé. Dans ce témoignage, Marie décrit les stratégies mises en place par sa fille pour «faire illusion» lorsqu'elle ne parvient pas à suivre une conversation : « elle fait souvent illusion $\downarrow$ c'est-à-dire que des fois elle comprend / des fois elle ne comprend pas mais elle fait comme si ».

Les stratégies d'illusion, de même que l'expectative de normalité - « les gens qui la voient comme ça / sans voir les appareils pensent qu'elle n'est pas sourde »-nous amènent à proposer une analyse sociolinguistique comparative entre discours sur la réhabilitation auditive en contexte de surdité et discours sur les langues minorisées, tous deux emprunts d'une idéologie de la langue standard. En effet, bien que les contextes divergent en ce sens que l'un se concentre sur une réalité strictement biomédicale et l'autre sur l'affirmation d'une norme linguistique fantasmée (Moreau, 1997) considérée comme la forme légitime, ces deux types de discours produisent un même sentiment d'insécurité linguistique et une véritable quête de reconnaissance sociale au sens de Goffman (1974). Dans La présentation de soi (1973), Goffman présente ainsi l'interaction comme un lieu semblable à une pièce de théâtre. Les participants à cette pièce de théâtre se définissent dans ce cadre comme des acteurs mettant en jeu leur image sociale dans une interaction donnée. La quête de reconnaissance pousse chaque

\footnotetext{
${ }^{5}$ L'implant cochléaire, expérimenté à partir des années 1980, consiste en une opération chirurgicale destinée à implanter un système artificiel dans l'oreille interne afin d'exciter électriquement les faisceaux de fibres du nerf cochléaire (Virole, $2006: 367$ ).
} 
«acteur » à mettre en avant les aspects de son identité les plus aptes à attirer la sympathie, l'admiration, l'estime, mais également à cacher les aspects pouvant être interprétés comme des défauts ou des faiblesses (Goffman, 1973). Cette stratégie d'illusion s'accompagne pour Élise d'une stratégie de camouflage de sa surdité et par le rejet de ce que nous nommons les « signes extérieurs de la surdité ». Jusqu'à récemment en effet, Élise refusait l'utilisation de la langue française parlée complétée ${ }^{6}$ (dés. code LPC) en classe, que ses parents l'encouragent à apprendre et à s'approprier.

Marie : « elle ne décode pas très bien non plus en fait $\downarrow$ ça a été assez long à mettre en place / ce n'est que depuis cette année que la codeuse nous dit que ça va mieux mais jusqu'à l'année dernière c'était "je me cache / je n'ai pas besoin / j'entends super bien je suis comme vous je n'ai pas besoin" $\downarrow »$.

Le témoignage présenté rend compte de l'évolution d'Élise dans l'acceptation de sa surdité et $\mathrm{du}$ cheminement familial dans l'accompagnement du processus de construction et d'affirmation identitaire d'un enfant sourd de parents entendants. Le discours d'Élise dévoile également la dimension invisible de la surdité (Goffman, 1975). Les signes extérieurs appareillages ; modalités linguistiques visuelles ; « accent sourd »-rendent visible la surdité d'Élise qu'elle entreprend temporairement de camoufler par le refus d'une communication en LPC et l'espoir de retirer un jour ses appareils :

Marie : « aujourd'hui elle se dit "pourquoi je suis sourde $\uparrow$ et quand je serai grande comme vous je n'aurai pas d'appareil $\uparrow$ je n'aurai pas d'implant" $\uparrow »$.

Marie : « elle a eu des remarques à l'école "pourquoi tu as des appareils" $\uparrow$ donc au début elle disait "parce que je n'entends pas très bien" $\downarrow$ elle avait du mal à dire le mot / et puis on a travaillé un peu avec elle / on a posé les choses / donc maintenant elle dit "je suis sourde" mais il n'y a pas plus tard que deux trois mois / elle me dit en larmes "maman // quelqu'un m'a dit que j'étais handicapée" $\downarrow$ ».

L'expérience d'Élise illustre le trouble provoqué par le questionnement lié au rapport flottant entre handicap et surdité. La surdité devient « un puissant marqueur social et prend part, en tant que caractéristique socio-identitaire, aux relations que [celle-ci] entretient avec son environnement » (Daure, Salaün, 2017 : 22). Par son caractère invisible, elle brouille les repères identitaires (Ibid.) et met en lumière la complexité de la notion d'identité - revendiquée par la communauté Sourde - «dans un mouvement constant de recherche de similitudes et de désir de différenciation (Marc, $2005: 11) »$.

Les stratégies d'illusion et de camouflage développées par Élise seraient ici finalement des mécanismes de différenciation à la surdité perçue en tant que handicap. Le témoignage que nous présentons amène à une complexification indispensable de la dichotomie entre surdité considérée d'un point de vue déficitaire et surdité revendiquée d'un point de vue anthropologique dans un mouvement constant d'assimilation et de différenciation (Marc, 2005) La surdité fait ainsi émerger une réflexion autour des multiples facettes que recouvre la notion d'identité, dans ses rapports interpersonnels et les représentations sociales qu'elle véhicule en contexte de surdité.

Caractérisée en tant que handicap dans la législation, la surdité fait l'objet d'approches radicalement différentes en ce qui concerne l'identité. Employée pour défendre l'existence d'une identité Sourde, cette notion est rarement définie et fréquemment posée comme principe fondateur d'une approche anthropologique de la surdité. En 1987, Gallissot proposait de

\footnotetext{
${ }^{6}$ La Langue française Parlée Complétée combine l'information visuelle de la lecture labiale à des informations manuelles positionnées à divers endroits autour du visage et délivre une information à propos des syllabes et des phonèmes de la langue parlée (Leybaert, 2012).
} 
substituer à la notion générale d'identité, celle d'identification, ouvrant ainsi la possibilité d'une réflexion sur la diversité des situations vécues par les sourds et leurs familles.

\section{L'identité et les identifications comme marqueurs de l'altérité}

L'identité est toujours mouvante et incertaine (De Mijolla, 2004). Censée définir un sujet, elle ne peut qu'être composé de « fragments » (Ibid., 2004). Dans la définition de l'identité Sourde pourtant, «être Sourd» ne constitue pas une identité fragmentée, mais l'affirmation d'une identité totale et objective où l'appartenance au groupe social des Sourds est « la première et la plus fondamentale de toutes les appartenances sociales, celle dans laquelle se nouent les liens les plus déterminants, parce qu'il s'agit de liens fondés sur une généalogie commune » (Cuche, 2001: 85) ${ }^{7}$. Smith définit "l'ethnie» par un nom propre en commun, le mythe d'une ascendance commune, une mémoire historique partagée, les éléments d'une culture en commun comme la langue ou la religion, les liens avec un pays d'origine (Smith, 1999). Des travaux menés en anthropologie par Siran mentionnent quant à eux l'idée d'une ethnie Sourde, associant la communauté Sourde à une ethnie non territorialisée, mais existant « au sein d'un monde la dominant $»$ :

Il n'est pas impossible qu'un chercheur de terrain rencontre à l'avenir une nouvelle ethnie dont on n'aurait guère qu'entendu parler ou qui n'aurait peut-être pas encore été reconnue pour telle. Un nouveau continent: l'événement serait plus considérable. Et pourtant! [...] Une nouvelle ethnie, voici qu'un certain nombre de travaux récents commencent à en décrire une: les Sourds. Une ethnie qu'il serait tout à fait impossible de territorialiser, encore moins d'isoler, et qui, tout comme celle des Manouches, serait immergée au sein d'un monde qui la domine et lui manque singulièrement de respect, mais dans lequel elle ne se dilue pas. [...] Existe-t-il une ethnie sourde? Oui. (Siran, 2004 : 173).

L'identité Sourde est caractérisée en tant que propriété inhérente au groupe de Sourds. En ce sens, la réponse de Pierre, sourd de parents entendants de 28 ans est éclairante, lorsque je lui pose la question : «qu'est-ce que l'identité Sourde pour toi ? » :

Pierre : « pour moi - l'identité Sourde c'est / d'abord / qu'est-ce qu'un vrai sourd $\uparrow$ c'est naitre sourd ça c'est la première chose $\downarrow$ deuxièmement c'est avoir une culture / une histoire qui est réelle $\downarrow$ ça c'est mon monde $\downarrow$ ».

L'appartenance fondamentale au groupe des Sourds est revendiquée : «Ça c'est mon monde ». Plus encore, c'est la mise en avant d'une généalogie commune " c'est naitre sourd» et même comme en témoigne l'ouvrage de Delaporte en 2002, appartenir à une lignée Sourde : un vrai sourd « est sourd de naissance, ses parents sont sourds, ses frères et sœurs sont sourds, il a une identité forte : c'est un vrai sourd [...]» (Delaporte, $2002: 180$ ).

La faible importance numérique des familles sourdes contraste avec leur poids culturel. Aux yeux des autres sourds, elles constituent une élite privilégiée, une sorte d'aristocratie. La langue et la culture des sourds s'y transmettent de génération en génération, exactement comme dans n'importe quelle culture humaine. (op. cit. : 169).

Pierre revendique l'opposition de l'identité Sourde au groupe des entendants et y fait référence :

\footnotetext{
${ }^{7}$ Denys Cuche fait ici notamment référence aux travaux de Geertz (1963). Voir l'article de Geertz Clifford, « The Integrative Revolution. Primordial Sentiments and Civil Politics in the New Sates », dans ID (éd.), 1963, Old Societies, New States, The Free Press, New York.
} 
Pierre : «il y a le «monde des Sourds » et il y a le «monde des entendants» $\downarrow$ c'est une expression mais pour moi c'est un deuxième monde $\downarrow$ mon vrai monde c'est le monde des Sourds $\downarrow$ l'art Sourd -1 'Histoire et son évolution ${ }^{8} \downarrow$ le chant signe et d'autres domaines aussi $\downarrow$ moi / aujourd'hui je suis militant du monde Sourd - je me bats parce que je ne veux pas que l'héritage disparaisse $\downarrow$ je veux continuer à transmettre cet héritage pour l'avenir $\downarrow$ ».

Les références à l'héritage et à l'Histoire des Sourds sont déterminantes dans la définition que donne Pierre de l'identité Sourde. L'identité Sourde est d'abord déterminée en tant qu'essence, «naitre sourd », par opposition aux personnes « devenues sourdes ». Pour Pierre, l'identité Sourde se constitue en opposition avec l'identité entendante : il y a le monde des sourds et il y a le monde des entendants. L'identité entendante n'existe par ailleurs qu'à travers la revendication d'une identité Sourde. Plus tard dans l'entretien, Pierre propose pourtant une alternative à ce clivage. Il propose de déplacer la problématique du «monde Sourd » et du « monde entendant» vers une approche considérant le «monde des signes » et «monde oral». Cette approche permet selon lui de décloisonner les catégories essentialistes que constitue la dichotomie Sourds et entendants en focalisant la distinction sur la modalité linguistique, accessible à tous. Pierre justifie cette appellation par le caractère discriminatoire qu'impose selon lui les catégorisations sourd et entendant :

Pierre : « tu vois c'est ça le problème $\downarrow$ il faut changer d'angle de vision $\downarrow$ j'ai beaucoup réfléchi en observant les gens $\downarrow$ si on enlève les termes "monde Sourd" et "monde entendant" // on efface ces expressions et on les remplace par "monde des signes" et "monde oral" $\downarrow$ tu vois c'est plus neutre $\downarrow$ il y a moins de limite parce que si on pense à monde Sourd / c'est limité $\downarrow$ ça exclut les entendants / ça veut dire aucun entendant $\downarrow$ c'est raciste $\downarrow$ pour moi c'est clairement raciste $\downarrow$ on exclut les entendants $\downarrow$ je préfère changer de regard et parler plutôt de monde des signes $\downarrow$ ça veut dire que le niveau on s'en fiche / tout le monde peut en faire partie à partir du moment où il connait la langue des signes $\downarrow$ c'est ça l'accessibilité $\downarrow$ on ne limite pas aux sourds mais à tous ceux qui signent $\downarrow$ ».

Par son témoignage, Pierre renverse l'idée d'accessibilité et par là même de handicap attribué aux sourds dans un monde d'entendants. Dans le «monde des Sourds », les entendants sont porteurs du handicap infranchissable de n'être pas. Ce changement de dénomination fait évoluer le critère essentialiste, indépassable, vers un critère linguistique, "accessible » par l'apprentissage de la langue des signes.

Cette réflexion de Pierre nous amène à déplacer la notion de l'identité vers celle de l'identification, qui offre un éclairage supplémentaire concernant les jeux de tension visibles pesant sur « monde Sourd » et le « monde entendant » et dont il est fréquemment question dans les discours portant sur la revendication de l'identité Sourde.

Dans son ouvrage La notion de culture dans les sciences sociales (2001), Denys Cuche rappelle la variabilité de la notion d'identité : «Il n'y a pas d'identité en soi, ni même uniquement pour soi. L'identité est toujours un rapport à l'autre. Autrement dit, identité et altérité ont partie liée, et sont dans une relation dialectique. L'identification va de pair avec la différenciation » (Cuche, 2001 : 87). La notion d'identification, proposée par Gallissot en 1987 et portée à notre connaissance par l'ouvrage de Denys Cuche (2001) permet ainsi de rappeler la relativité du concept d'identité qui ne fonctionne que dans la relation et dans une dialectique sourdsentendants: "Dans la mesure où l'identité est toujours la résultante d'un processus d'identification au sein d'une situation relationnelle, dans la mesure aussi où elle est relative, car elle peut évoluer si la situation relationnelle change, sans doute vaudrait-il mieux retenir

\footnotetext{
${ }^{8}$ De même que nous adoptons la graphie Sourd en majuscule, nous associons l'Histoire Sourde en majuscule à ce mouvement de revendication culturelle. L'Histoire Sourde fait référence aux événements fondateurs de la communauté Sourde.
} 
comme concept opératoire pour l'analyse celui d'“identification" plutôt que celui d'identité » (Gallissot, 1987 : 27). Ainsi, « l'identification, qui est l'un des aspects de la quête du semblable, sous-tend l'identité ; mais tout aussi importante est l'aspiration à la distinction, composante nécessaire du sentiment d'individuation »(Marc, 2005: 11). Les relations sociales sousjacentes à la notion d'identité Sourde sont exprimées dans les discours portant sur les Sourds (Delaporte, 2002 ; 2010 ; Meynard, 2010 ; Holcomb, 2016 ; Bédoin, 2018), mais rarement mises en avant. Ces relations au groupe entendant évoluent avec le temps : les mouvements de revendication pour la reconnaissance d'une identité Sourde et d'une culture propre prennent leur essor dans les années soixante-dix en France. À cette période, la langue des signes n'est pas reconnue comme langue à part entière ; la loi de $1991^{9}$ permettant une liberté de choix dans l'éducation des élèves sourds n'a pas été votée et malgré le décalage entre les textes de loi et leur mise en application effective, le regard porté sur les sourds et la langue des signes n'a eu de cesse d'évoluer : le développement des cours de langue des signes proposés en France et le renversement du profil des apprenants de langue des signes, qui est aujourd'hui constitué, pour une large majorité, des personnes entendantes en sont des exemples significatifs.

Parler d'identification au groupe des Sourds plutôt que d'identité Sourde permet de rappeler ce lien étroit au groupe des entendants, existant uniquement par ce rapport : "L'identification procède des relations sociales immédiates et fait référence à un imaginaire communautaire (identification par projection collective)» (Gallissot, 1987: 27). Les Sourds sont immédiatement confrontés aux entendants, un groupe qui n'existe que par les discours autour de la surdité pour permettre l'affirmation de l'identité Sourde. La notion d'identification permet en cela de rendre visibles les positionnements des Sourds concernant les revendications de leur altérité et de leur singularité. Cette visibilité écarte la confusion d'un terme - l'identité -, dont l'impensé amènerait, d'une part, à l'effacement progressif de la relation à l'autre, pourtant primordiale pour comprendre les discours et les enjeux liés à la langue des signes et l'appartenance à un groupe minoritaire et minorisé, et d'autre part à l'effacement de l'identité réelle.

L'identité « réelle », introduite par Georges Devereux en 1972, révèle une distance entre ce qu'il nomme "l'identité ethnique», ici, l'identité Sourde et «l'identité réelle», celle qui distingue tout être humain : "Pour avoir une identité ethnique, on doit être humain pour commencer. Or la qualité de l'être humain implique précisément la capacité d'être hautement unique, hautement différent des autres »(Devereux, 1972:132). Devereux rappelle ainsi l'importance de l'individu, unique en soi, avant d'être le membre d'une classe ou d'un groupe.

Si pour Pierre, l'identité Sourde, c'est d'abord «naitre sourd » et ensuite « avoir une culture et une histoire qui est réelle », pour de nombreux parents entendants interrogés, le fait que leur enfant soit né sourd ne constitue généralement pas en soi une identité Sourde, quand bien même ces derniers auraient effectué un choix linguistique en faveur de la langue des signes. Lucie a fait le choix avec son compagnon d'un parcours linguistique vocal et en langue des signes pour leur fils Gabriel, qui a six ans au moment de notre entretien en 2017 et qui est implanté. Notre échange porte sur son vécu relatif à la notion d'identité Sourde.

Lucie : « il a développé une identité d'un petit garçon / sourd / implanté ${ }^{10} \downarrow$ après // il est sur une identité // qui / je pense ressemble à celle de ses copains à [nom de l'institut médico-social] / de sourds implantés $\downarrow$ ».

Lucie : « est-ce qu'il développe une identité Sourde $\uparrow$ est-ce qu'il a conscience d'être sourd $\uparrow$ ça c'est sûr $\downarrow$ ».

\footnotetext{
${ }^{9} \mathrm{https}: / /$ www.legifrance.gouv.fr/affichTexte.do?cidTexte=JORFTEXT000000535308\&dateTexte ; https://www.legifrance.gouv.fr/affichTexte.do?cidTexte=JORFTEXT000000710255\&categorieLien=id

${ }^{10}$ Les parents de Gabriel ont fait le choix de l'implantation cochléaire pour leur fils.
} 
« Naitre Sourd» et «avoir une culture et une histoire réelle ${ }^{11}$ ne constituent pas ici, en eux-mêmes, une identité Sourde. Lucie met en avant l'idée d'une identité nécessairement fragmentée (De Mijolla, 2004), qui constituent l'identité réelle (Devereux, 1972) de son fils, qui avant même d'être sourd est un petit garçon avec des amis qui lui ressemblent, sourds et implantés. Elle nous amène par ailleurs, à travers son témoignage, à repenser la question de l'identité Sourde autour de la conscience de sa propre altérité : « est-ce qu'il a conscience d'être sourd?». Gabriel n'est pas que sourd, son identité est d'abord caractérisée par son individualité, par le fait qu'il est, en plus d'un petit garçon, un petit garçon sourd. La notion d'identité (« de classe » pour Devereux) renvoie aux marqueurs hiérarchisables de l'individu, c'est-à-dire ce qui prime dans l'identification. La réflexion que nous menons engage en ce sens vers un positionnement en faveur des notions d' « identifications » ou encore de « marqueurs ». L'identité en tant qu'essence de la personne, comporte le danger de "limiter l'identité à la catégorisation "d'être(s)" " (Tap, 2016: 46). Nous rappelons le décalage des situations parentales et individuelles, entre des parents entendants pour qui la langue des signes est généralement une langue méconnue, et une partie des membres de la communauté Sourde (parmi la diversité des conceptions de la surdité également présentes au sein de cette communauté), revendiquant la langue des signes comme moyen exclusif de faire apparaitre la "véritable" identité des personnes sourdes. "Au niveau pratique, ces discordances sont souvent dues à des tentatives de représenter la personnalité ethnique - ce qu'elle "est" - comme conforme à l'identité ethnique qui, elle, dans de tels cas, est traitée comme un "devrait être" : comme un modèle idéal de comportement, ce que, logiquement, elle n'est pas ou n'est pas en premier lieu » (Devereux, 1972 : 136). Par ces discours d'appartenance à un autre groupe que le leur, certains des parents rencontrés relatent la violence ressentie face au jugement porté sur leurs trajectoires familiales et choix linguistiques :

Isabelle : «on nous a dit / c'est vrai que // comment dire / la surdité il faut qu'elle // il faut l'intégrer / il faut l'accepter mais on nous a dit qu'on ne l'acceptait pas en mettant des appareils à nos enfants $\downarrow$ les sourds signeurs // qui vous renvoient ça $\downarrow$ ».

Enquêtrice : « vous en avez côtoyé $\uparrow$ ».

Isabelle : « on en a côtoyé [des sourds] par l'intermédiaire du [nom du centre médico-social] / qui a pu organiser quelques réunions où tout le monde essayait d'aller// on n'était pas fermés $\downarrow$ mais punaise qu'est-ce qu'on s'en est pris dans la tronche $\downarrow$ moi je ressortais de là en pleurs $\downarrow$ parce qu'on nous renvoyait qu'on n'avait pas accepté nos enfants tels qu'ils étaient / qu'on voulait les transformer $\downarrow$ ça c'est dur $\downarrow / /$ ça c'est dur $\downarrow$ ».

Là où l'identité Sourde est revendiquée comme l'appartenance à un groupe « réel », les discours essentialistes peuvent être vécus comme une violence pour des parents accusés de ne pas « accepter » leur enfant et de vouloir le « transformer ». La convocation de l'identité Sourde de leur enfant et d'une filiation essentialiste est subie par les parents entendants et doit interpeller sur une conception autoristariste du « même » et de «l'autre » (De Mijolla, 2004). L'identification exclusive par l'essence et l'ethnicisation de la surdité ont pour effet de brouiller les liens de filiation entre parents entendants et enfants sourds. Le témoignage d'Isabelle met en lumière un surinvestissement de cette notion d'identité « qui conduit, de fait, à une réduction des identités de classe significatives que l'on possède, à une seule - et donc à l'anéantissement de l'identité réelle des individus » (Devereux, 1972).

\footnotetext{
${ }^{11}$ Nous reprenons ici les propos de Pierre.
} 
Des parents témoignent des enjeux et des discours portants sur les sourds implantés et de leurs craintes quant à l'entrave que le choix d'implantation qu'ils ont effectué pourrait avoir dans l'accès à la communauté Sourde pour leur enfant :

Carine : «si mon fils il a besoin de signer un jour / ou d'aller dans le monde des sourds signants / il ira $\downarrow$ s'il a besoin d'aller se confronter à ça / il ira et on ne va pas le rejeter $\downarrow$ et j'espère qu'il ne sera pas rejeté parce qu'à un moment donné il a eu un implant ».

Ce témoignage d'une mère entendante est révélateur d'une certaine culpabilisation des parents et de la distance de ces derniers pour le monde Sourd qu'ils décrivent généralement de loin, ne s'y sentant pas légitimes, à moins d'embrasser intégralement les représentations liées à l'identité Sourde. Cette mère ne s'offre pas la possibilité d'aller à la rencontre de ce monde duquel elle sait être par essence autre et craint que son fils ne soit autorisé à y entrer, " à cause » du choix d'implantation que ses parents ont fait pour lui. La lecture de ce témoignage peut également s'effectuer au prisme d'un refus des parents d'aller à la rencontre de la communauté Sourde par manque d'intérêt (Holcomb, 2016). Pour Lhéricel (2006), l'exclusion des parents entendants pourrait ainsi expliquer les réticences à s'engager vers une modalité linguistique en langue des signes. Les enfants développant de plus en plus une communication en langue des signes et s'intéressant de plus en plus à une culture dans laquelle leurs parents « n'auraient pas leur place » ces derniers peuvent avoir le sentiment que leur enfant leur échappe :

Le fait que leur enfant se tourne et se dirige vers la langue signée ne représenterait-il pas une coupure, un danger de changement identitaire tel que l'enfant ne serait plus réellement leur enfant, mais aurait plutôt tendance à leur échapper "à cause" de cette langue et ce qu'elle entraine avec elle? Aux yeux des parents, l'enfant quitterait alors définitivement le "monde des entendants" pour rejoindre celui des sourds, une sorte d'aller simple sans retour, dans lequel eux n'auraient pas leur place. (Lhéricel, 2006 : 30).

\section{Conclusion}

Les entretiens et analyses que nous avons présentés à travers cet article offrent un regard nouveau sur des trajectoires parentales complexes, peu décrites dans la littérature scientifique actuelle et qui pourtant représentent la part extrêmement majoritaire des situations familiales dans lesquelles les enfants sourds naissent. Rendre compte du parcours d'un petit nombre de parents entendants, à partir des premiers doutes, amorcés aujourd'hui en France par la mise en place systématique du dépistage néonatal de surdité en maternité, jusqu'aux choix de langue et de scolarisation, suscite des réflexions et questionnements que nous avons souhaité ouvrir à la lumière de différents axes ${ }^{12}$, ici la question de l'identification à une communauté et une langue généralement méconnue par les parents entendants.

Par le croisement des témoignages de parents entendants et de personnes sourdes adultes, nous avons souhaité mettre en lumière des situations de tensions et de mécompréhensions, qui amènent à repenser les notions d'identité et de communauté Sourde, ou tout du moins à faire émerger une réflexion, que nous considérons impérative, sur la place des parents entendants au sein de ces discours.

\footnotetext{
${ }^{12}$ Pour une réflexion autour de la question du dépistage néonatal de surdité, à travers le vécu de parents entendants en France, nous renvoyons le lecteur à l'article Rannou, 2017.
} 


\section{Bibliographie}

BÉDOIN D., 2018, Sociologie du monde des sourds, La Découverte, Paris, 128 pages.

CUCHE D., 2001 [1996], La notion de culture dans les sciences sociales, La Découverte, Paris, 160 pages.

DAURE I, SALAÜN F., 2017, « Le handicap invisible... ou le décalage entre ce qui se voit et la réalité », dans Le Journal des psychologues n³48 (6), pp.22-27

DELAPORTE Y., 2002, Les sourds c'est comme ça, Édition de la Maison des sciences de l'homme, Paris, 398 pages.

DELAPORTE Y., 2010 [2000], « Être sourd dans un monde entendant: destin, rencontre, transmission et rupture », Ethnologie française n³, pp. 389-400.

DEVEREUX G., 1972, Ethnopsychanalyse complémentariste, Flammarion, Nouvelle bibliothèque scientifique, Paris, 282 pages.

GALLISSOT R., 1987, "Sous l'identité, le procès d'identification », L'homme et la société $n^{\circ} 83$, L’Harmattan, Paris, pp. 12-27.

GOFFMAN E., 1973, La Présentation de soi. Mise en scène de la vie quotidienne 1, Éditions de Minuit, Paris, 256 pages.

GOFFMAN E., 1974, Les rites d'interaction, Éditions de Minuit, Paris, 240 pages.

HOLCOMB T., 2016, Introduction à la culture sourde, Érès, Paris, 451 pages.

LEYBAERT J. (dir.), 2012, La langue française parlée complétée (LPC). Fondements et perspectives, De Boeck, Bruxelles, 270 pages.

LHÉRICEL B., 2006, « Du choix de la langue pour l'enfant sourd » in Daigle D., Parisot, A.-M., Surdité et Société : Perspectives Psychosociale, Didactique et Linguistique, Presses de l'université du Québec, Montréal, pp. 27-41.

MARC E., 2005, Psychologie de l'identité. Soi et le groupe, Dunod, Paris, 255 pages.

MEYNARD A., 2010, Soigner la surdité et faire taire les Sourds. Essai sur la médicalisation du Sourd et de sa parole, Érès, 326 pages.

MOREAU M.-L. (dir.), 1997, Sociolinguistique : les concepts de base, Éditions Mardaga, Bruxelles, 312 pages.

PADDEN C., 1980, "The deaf community and the culture of deaf people", In Baker C., Battison R. (Eds.), Sign language and the deaf community, Silver Spring, National Association of the Deaf, Washington D.C, pp. 89-104.

RANNOU P., 2017, « Parents entendants d'enfants sourds en France : récits de mères illustrant les écarts entre discours officiels et pratiques des professionnels face à la diversité des modèles de communication existants ", Alterstice, volume 7, numéro 2, pp. 67-76, [en ligne], $\quad<$ https://www.erudit.org/fr/revues/alterstice/2017-v7-n2alterstice04033/1052570ar.pdf>, consulté le 20 février 2020.

RANNOU P., 2018, Sociolinguistique de la surdité, didactisation de la pluralité linguistique. Parcours de parents entendants en France et regards croisés sur la scolarisation des élèves sourds : France-États-Unis, doctorat en Sciences du langage, sous la direction de Gudrun Ledegen et Ye Wang, Université Rennes 2, Columbia University in New York City, 625 pages.

SMITH A., 1998, Nationalism and modernism : a critical survey of recent theories of nations and nationalism, Routledge, Londres, 270 pages.

SIRAN J.-L., 2004, « Des Sourds », L’Homme n¹69, EHESS, Paris, pp. 173-185.

STOKOE W., CASTERLINE D., CRONEBERG C., 1965, A dictionary of American Sign Language on linguistic principles, Silver Spring, Linstok Press, Washington D.C, 346 pages.

TAP P., 2016, "Marquer sa différence (Entretien avec Pierre Tap », dans Identité (s). L'individu, le groupe, la société, Éditions Sciences Humaines, pp. 46-50. 


\section{GLOTTOPOL}

Revue de sociolinguistique en ligne

Comité de rédaction : Michaël Abecassis, Salih Akin, Sophie Babault, Claude Caitucoli, Véronique Castellotti, Régine Delamotte, Robert Fournier, Stéphanie Galligani, Emmanuelle Huver, Normand Labrie, Foued Laroussi, Benoit Leblanc, Fabienne Leconte, Gudrun Ledegen, Danièle Moore, Clara Mortamet, Alioune Ndao, Isabelle Pierozak, Gisèle Prignitz.

Rédactrice en chef : Clara Mortamet.

Comité scientifique : Claudine Bavoux, Michel Beniamino, Jacqueline Billiez, Philippe Blanchet, Pierre Bouchard, Ahmed Boukous, Pierre Dumont, Jean-Michel Eloy, Françoise Gadet, Monica Heller, Caroline Juilliard, Jean-Marie Klinkenberg, Jean Le Du, Marinette Matthey, Jacques Maurais, Marie-Louise Moreau, Robert Nicolaï, Didier de Robillard, Paul Siblot, Claude Truchot, Daniel Véronique.

\section{Comité de lecture pour ce numéro :}

Mickael Abecassis, Michelle Auzanneau, Salih Akin, Céline Amourette, Gabriel Bergounioux, Philippe Boula de Mareuil, Catherine Brissaud, Aude Bretegnier, Maria Candea, Véronique Castellotti, Régine Delamotte, Jean-François De Pietro, Catherine Delarue-Breton, Anne Dister, Alexandre Duchêne, Valentin Feussi, Françoise Gadet, Stéphanie Galligani, François Gaudin, Monica Heller, Emmanuelle Huver, Caroline Juilliard, Abou Bakri Kebe, Jean-Marie Klinkenberg, Christian Lagarde, Foued Laroussi, Fabienne Leconte, Evelyne Lloze, Nolwenn Lorenzi, Gudrun Ledegen, Nadja Maillard, Marinette Matthey, Bruno Maurer, Véronique Miguel Addisu, Saskia Mugnier, Claire Oger, Marielle Rispail, Richard Sabria, Véronique Traverso, Christel Troncy, Cécile Van den Avenne. 